\title{
How to Design U1 snRNA Molecules for Splicing Rescue
}

\author{
Liliana Matos, Juliana I. Santos, Ma . Francisca Coutinho, \\ and Sandra Alves
}

\begin{abstract}
Mutations affecting constitutive splice donor sites $\left(5^{\prime} \mathrm{ss}\right)$ are among the most frequent genetic defects that disrupt the normal splicing process. Pre-mRNA splicing requires the correct identification of a number of cis-acting elements in an ordered fashion. By disrupting the complementarity of the 5 'ss with the endogenous small nuclear RNA Ul (Ul snRNA), the key component of the spliceosomal Ul ribonucleoprotein, $5^{\prime}$ ss mutations may result in exon skipping, intron retention or activation of cryptic splice sites. Engineered modification of the Ul snRNA seemed to be a logical method to overcome the effect of those mutations. In fact, over the last years, a number of in vitro studies on the use of those modified Ul snRNAs to correct a variety of splicing defects have demonstrated the feasibility of this approach. Furthermore, recent reports on its applicability in vivo are adding up to the principle that engineered modification of Ul snRNAs represents a valuable approach and prompting further studies to demonstrate the clinical translatability of this strategy.

Here, we outline the design and generation of Ul snRNAs with different degrees of complementarity to mutated 5'ss. Using the HGSNAT gene as an example, we describe the methods for a proper evaluation of their efficacy in vitro, taking advantage of our experience to share a number of tips on how to design U1 snRNA molecules for splicing rescue.
\end{abstract}

Key words Ul snRNA-based therapy, Splicing modulation, 5'ss mutations, Aberrant exon skipping, Modified Ul snRNA, Mucopolysaccharidosis IIIC

1 Introduction

The Ul small nuclear ribonucleoprotein (Ul snRNP) is a key molecule involved in an early event of the splicing process. Like other snRNPs involved in the overall splicing regulation process, it contains a small RNA complexed with several proteins, namely seven Smith antigen $(\mathrm{Sm})$ proteins and three Ul-specific proteins (UlA, UlC, and Ul70K) [1]. Ul snRNA, the RNA component of the Ul snRNP is a 164 nucleotides-long molecule whose $5^{\prime}$ end interacts by complementarity with the $5^{\prime}$ splice donor site $\left(5^{\prime}\right.$ ss $)$. That interaction between the single stranded $5^{\prime}$ tail of the Ul snRNA molecule and the moderately conserved stretch of nucleotides that constitutes the $5^{\prime}$ ss (CAG/GURAGU, where $\mathrm{R}$ is a 
purine) marks the exon-intron boundary and initiates spliceosome assembly [2]. About $40 \%, 22 \%$, and $5 \%$ of normal 5 'ss contain two, three, or four mismatches towards the Ul snRNA, respectively $[3,4]$. This variable degree of degeneration is among the major factors that significantly contribute to hinder a clear prediction of the effect of mutations flanking the canonical GU site. Furthermore, there is a number of additional elements, which may influence the splice site selection and need to be taken into account such as splicing silencer and enhancer motifs, the presence of alternative splice sites, secondary structures, and regulatory proteins [5]. Therefore, a straightforward prediction of the effect of mutations flanking the canonical GU site without a direct assessment of the mature mRNA produced can be quite challenging. Interestingly, however, it is also the variable degree of degeneration of $5^{\prime}$ ss and the surprising heterogeneity existing among human spliceosomal snRNA, which allows for splicing correction using modified exogenous Ul snRNAs.

Overall, the rationale on the use of modified Ul snRNAs to correct splicing defects is as simple as it can be: as $5^{\prime}$ ss mutations alter the $5^{\prime}$ ss recognition by the endogenous Ul snRNA, exogenous Ul snRNAs may be engineered through complementary base pairing in order to correctly recognize the mutated allele and initiate spliceosome assembly, thus suppressing the mutation effect.

So far, the effects of modified Ul snRNAs have been tested in vitro in a number of cellular platforms from patient-derived cells to model cell lines overexpressing the splicing defects under study, and their potential to either fully or partially correct those mutations was demonstrated for a number of different diseases $[5,6]$. Importantly, the application of this sort of modified Ul snRNAs in animal models has also been addressed in recent studies, with a few promising results reported to date [7-10] (see Note 1).

Globally, mutations affecting constitutive $5^{\prime}$ ss represent roughly $8 \%$ of all known genetic disease-causing variants. Their pathogenicity derives from the reduced complementarity of the Ul snRNA to the $5^{\prime}$ ss. $5^{\prime}$ ss mutations mostly result in exon skipping but their effect over splicing may vary. Currently, there are a number of in silico tools that may help predict disease-causing effects, but cDNA analysis remains mandatory for a proper assessment of their consequence over splicing. For example, mutations affecting RNA splicing represent more than $20 \%$ of the mutant alleles in Mucopolysaccharidosis type IIIC (MPS IIIC; HGSNAT gene), a rare lysosomal storage disorder that causes severe neurodegeneration. Many of these mutations are located in the conserved splice donor or acceptor sites, while few are found in the nearby nucleotides. For three mutations that affect the donor site, we have previously developed different modified Ul snRNAs with compensatory changes that may allow for proper recognition of the mutated $5^{\prime}$ ss, in an attempt to rescue the normal splicing process. 
A

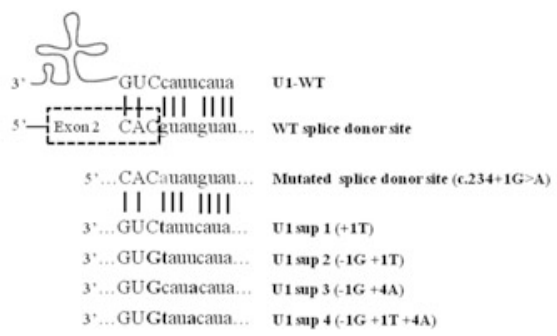

B
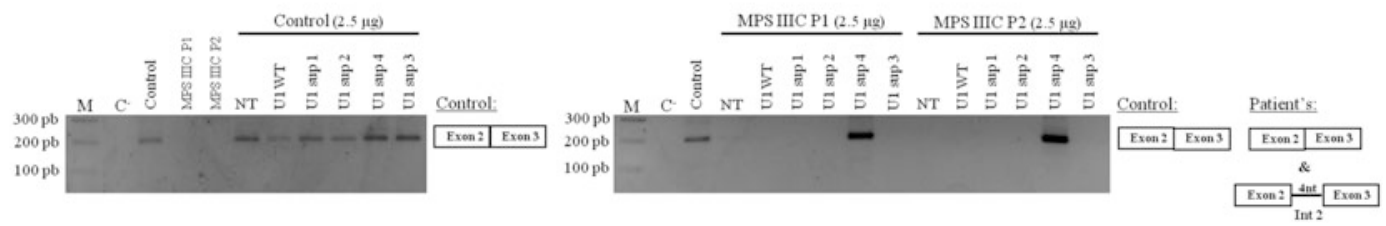

Fig. 1 Modified U1 snRNA therapeutic approach to correct the pathogenic effect of a $5^{\prime}$ splice site mutation on the HGSNAT gene. (a) Schematic illustration of base pairing between the wild-type U1 (U1-WT) and the 5'ss of wild-type and mutant exon 2 of the HGSNAT gene. The mutation position in the $5^{\prime}$ ss is marked in grey and it is in italics. The different U1 snRNAs used for the mutated 5'ss of HGSNAT (designated as U1-sup, for suppressor) are also shown. The U1 sequence modifications are illustrated in bold. (b) RT-PCR analysis of the endogenous splicing pattern of control and MPS IIIC patients derived fibroblasts after transfection with different $\mathrm{U} 1$ isoforms. The constitutive splicing of exon 2 of the HGSNAT gene was not altered in control fibroblasts after overexpression of U1-WT or any of the modified U1 constructs. In the MPS IIIC patients 1 (MPS IIIC P1) and 2 (MPS IIIC P2), bearing the homozygous mutation c.234+1G $>$ A, only the fully adapted U1 (U1-sup4) resulted in partial correction of exon 2 skipping

For the c.234+1G $>$ A mutation, a totally complementary Ul snRNA allowed for partial correction of exon 2 aberrant splicing in patients' fibroblasts (Fig. 1) [11]. Here, we take advantage of our experience on the development of modified Ul snRNAs to compensate for those HGSNAT mutations, to present a practical overview on how to design Ul snRNA molecules for splicing rescue.

In summary, we present an overview of the experimental design for in vitro testing the potential of modified Ul snRNA vectors to correct aberrant splicing caused by $5^{\prime}$ ss mutations. Briefly, we show: (a) how to design in silico Ul's with different degrees of complementarity to each mutated $5^{\prime}$ ss by introducing a number of sequence changes, and (b) how the different Ul vectors harboring those alterations are obtained by site-directed mutagenesis of the original wild-type (WT) human Ul snRNA-harboring pG3Ul vector [12], a derivative of pHUl [13]. We also describe how these molecules are transfected into patients' fibroblasts and how their effectiveness on splicing redirection can be assessed by posttransfection cDNA analysis and sequencing. Finally, we elaborate on the relevance of further addressing the treatment's effect at protein level. 


\section{Materials}

\subsection{Generating Modified U1 snRNA Vectors Adapted to the 5 ss of Interest}

1. The Homo sapiens Ul snRNA gene sequence is required to design primers for site-directed mutagenesis PCR and can be found in the Ensembl database (ENSG00000104852).

2. The sequence of the $5^{\prime}$ ss of interest for splicing rescue can be found in Ensembl or other reference sequence databases (in this particular chapter we used the Homo sapiens HGSNAT gene sequence, ENSG00000165102).

3. pG3Ul vector [12] a derivative of pHUl [13] (see Note 2).

4. Sense and antisense mutagenic primers.

5. PCR mutagenesis kit.

6. PCR thermocycler.

7. Chemically Escherichia coli competent cells (Homemade or commercial; usually are included in the PCR mutagenesis kits).

8. Water bath.

9. Thermomixer.

10. Ice.

11. Super optimal broth with catabolite repression (SOC) medium (commercially available).

12. Luria-Bertani (LB) agar medium (commercially available; sterilize by autoclaving) plates with selection antibiotic $(100 \mu \mathrm{g} /$ $\mathrm{mL}$, ampicillin; see Note 3 ).

13. Sterile bacterial cell spreaders.

14. Plasmid DNA miniprep purification kit.

15. LB liquid medium (commercially available; sterilize by autoclaving).

16. Ampicillin.

17. $15 \mathrm{~mL}$ conical centrifuge tubes.

18. Sterile tips.

19. Orbital shaking incubator.

20. pG3Ul forward primer (Ul-seq $\mathrm{Fw}_{-}-5^{\prime}$ CACGAAG GAGTTCCCGTG 3').

21. Sterile flasks (1 L).

22. Endotoxin-free maxiprep plasmid DNA purification kit.

23. $40 \%$ Glycerol (sterilize by autoclaving).

24. $2 \mathrm{~mL}$ polypropylene conical tubes. 
2.2 In Vitro

Therapeutic Evaluation

of Modified U1 snRNA

Vectors in Human

Fibroblasts

2.2.1 Transfection of

Modified U1 snRNA Vectors

in Human Fibroblasts
2.2.2 Analysis of Splicing

Rescue by RT-PCR
1. Human Dermal Fibroblasts from patients harboring the mutation under analysis (e.g. fibroblasts from patients' with MPS IIIC, carrying the c.234+lG $>$ A mutation in homozigosity) and WT Human Dermal Fibroblasts to use as control.

2. Dulbecco's Modified Eagle's Medium (DMEM) + Glutamax supplemented with 10\% Fetal Bovine Serum (FBS), 5\% penicillin/streptomycin (PenStrep) antibiotics, and 5\% amphotericin B (Fungizone ${ }^{\circledR}$ ).

3. Phosphate buffered saline $1 x$ (PBS).

4. Trypsin-EDTA.

5. $\mathrm{CO}_{2}$ incubator.

6. $15 \mathrm{~mL}$ conical centrifuge tubes.

7. Refrigerated centrifuge.

8. Neubauer chamber (hemocytometer).

9. Inverted Microscope.

10. Hand cell counter.

11. T-75 $\mathrm{cm}^{2}$ cell culture flasks.

12. 6-well cell culture plates.

13. Opti-MEM ${ }^{\mathrm{TM}}$ Reduced Serum Medium.

14. Transfection reagent.

15. 1.5 and $2 \mathrm{~mL}$ polypropylene conical tubes.

16. Modified Ul snRNA constructs (see Subheading 2.1).

1. RNA isolation kit.

2. Refrigerated centrifuge.

3. $1.5 \mathrm{~mL}$ polypropylene conical tubes.

4. Spectrophotometer for nucleic acids measurement.

5. cDNA synthesis kit.

6. Taq DNA polymerase.

7. Oligo $(\mathrm{dT})_{18}$ primer mix (if required).

8. Gene-specific primers (e.g. HGSNAT primers-Exon 2 Fw: 5' ACATGCAGAGCTGAAGATGGA 3'; Exon 3 Rv: 5' GATA GATCCGTGCTGGGTG 3').

9. Ice.

10. RNase free water.

11. PCR thermocycler.

12. Agarose gel with ethidium bromide for electrophoresis. 
13. DNA Ladder (molecular weight size marker).

14. UV transilluminator.

15. Sterile scalpel blades.

16. PCR products purification kits.

\section{Methods}

\subsection{Generating the Modified U1 snRNA Vectors}

To design the primers for producing the desired modified human Ul snRNA vectors, it is first necessary to know the sequences of the $5^{\prime}$ ss under study, both WT and mutant. Then, it is necessary to analyze the complementarity of those sequences with that of $\mathrm{Ul}$ snRNA. Next, several modified Ul snRNA vectors can be designed and constructed to have different complementarities to the target sequences (Fig. 2). To generate those constructs, the plasmid pG3U1 [12] (kindly provided by Prof. Dr. Belén Pérez) a derivative of pHUl [13], containing the coding sequence of the human Ul can be used as template for site-directed mutagenesis PCR reactions (see Note 2). Depending on the number of mutations to insert in the Ul snRNA vector sequence, different mutagenic primer pairs need to be designed.

A

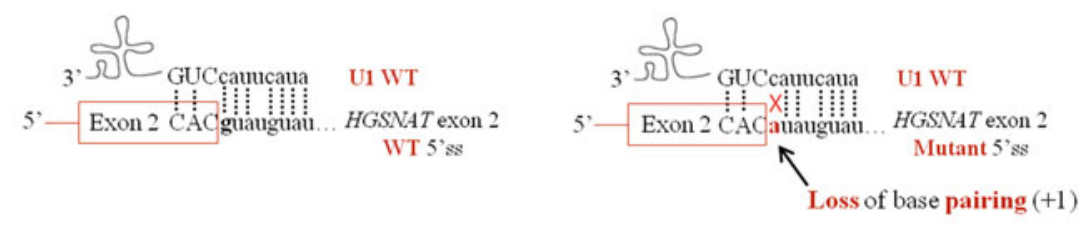

B

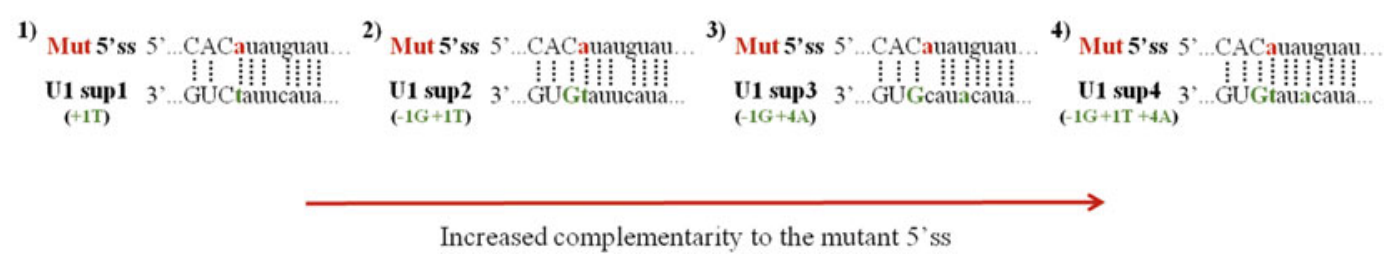

Fig. 2 Design and construction of modified U1 snRNA vectors. (a) Schematic representation of base pair interactions between the U1 snRNA and the wild-type and mutant 5'ss of HGSNAT exon 2, respectively. (b) Illustration of the strategy followed to increase the complementarity of U1 snRNA with the mutated $5^{\prime}$ ss of HGSNAT gene. U1 complementarity was increased stepwise, and to try to compensate for the HGSNAT mutation at +1 position, four different U1-adaptations were designed [U1 sup1 $(+1 \mathrm{~T})$; U1 sup2 $(-1 \mathrm{G}+1 \mathrm{~T})$; U1 sup3 $(-1 G+4 A)$; U1 sup4 $(-1 G+1 T+4 A)]$. Upper case letters show exonic nucleotides, whereas the lower case letters denote intronic nucleotides. Base pairing is indicated by vertical lines and its loss by an $X$. The mutant nucleotide is highlighted in red and the changed nucleotides in the U1 sequence are illustrated in green 
3.1.1 Engineering Modified U1 snRNA Vectors Adapted to the 5 ss of Interest
1. According to the different modifications to be introduced in the Ul snRNA vector sequence, design sense and antisense primers with the desired mutation(s) to be introduced by sitedirected mutagenesis (see Note 4).

2. Using the mutagenic primers, perform the site-directed mutagenesis of the WT Ul snRNA vector using the mutagenesis kit (see Note 5). Briefly, mix the Ul snRNA plasmid DNA ( 40 ng) with primers, buffer, dNTPs (according to the kit), apyrogenic water, a High Fidelity Taq polymerase and subject the mixture to recommended PCR conditions from the mutagenesis kit. The number of PCR cycles varies according to the type of the desired mutation(s) (see Note 6); and the number $(n)$ of min of the PCR extension step depends on the plasmid length, $n$ is calculated as $1 \mathrm{~min} / \mathrm{kb}$; see Note 7 . After the PCR reaction is completed, add $1 \mu \mathrm{L}(10 \mathrm{U})$ of $D p n \mathrm{I}$ restriction enzyme to the amplified products and incubate for $1 \mathrm{~h}$ at $37^{\circ} \mathrm{C}$ to digest the parental dsDNA.

3. Use $1-4 \mu \mathrm{L}$ of the $D p n \mathrm{I}$ treated DNA reaction to transform E. coli competent cells. Briefly, thaw on ice a $50 \mu \mathrm{L}$ aliquot of competent cells and add $1-4 \mu \mathrm{L}$ of the digested reaction. Swirl the tube gently to $\mathrm{mix}$ and incubate on ice for $30 \mathrm{~min}$. In a water bath or dry thermomixer, heat pulse the tube at $42{ }^{\circ} \mathrm{C}$ for $45 \mathrm{~s}$ and then place the reaction tube on ice for $2 \mathrm{~min}$. Add room temperature SOC medium $(5 \times$ the volume of competent cells) and incubate for $1 \mathrm{~h}$ with shaking at $600 \mathrm{rpm}$ in a dry thermomixer (see Note 8). After incubation spread the appropriate volume (see Note 9) of transformation reaction on pre-warmed $\left(37^{\circ} \mathrm{C}\right)$ LB-agar plates containing ampicillin $(100 \mu \mathrm{g} / \mathrm{mL})$ and incubate at $37^{\circ} \mathrm{C}$ for $16-18 \mathrm{~h}($ see Note 10$)$.

4. To obtain plasmid DNA minipreps, prepare minicultures of selected bacterial colonies to allow their growth. Add $3 \mathrm{~mL}$ (see Note 11) of LB medium containing ampicillin (100 $\mu \mathrm{g} /$ $\mathrm{mL}$ ) to a $15 \mathrm{~mL}$ tube and using a sterilized pipette tip pick a colony and add it into the medium by pipetting up and down (or, simply, place the pipet tip into the medium). Repeat the procedure for 3-5 colonies. Incubate the tubes in an orbital shaking incubator at $220 \mathrm{rpm}$ and $37^{\circ} \mathrm{C}$ for $16-18 \mathrm{~h}$. To purify the plasmid DNA prepare DNA minipreps using a plasmid miniprep purification kit (see Note 12). Select the mutant (s) Ul snRNA plasmid(s) by Sanger sequencing analysis (Ul-seq $\mathrm{Fw}_{\mathrm{w}}$ primer) using 100 ng of purified miniprep.

5. Once the desired modified Ul snRNA construct(s) are selected, propagate them in maxicultures to obtain a high quantity of the modified construct(s) that can be used for transfection. First, prepare a miniculture of each case according to step 4 ( see Note 13). Then add 100-150 mL of LB medium 


\subsection{In Vitro \\ Therapeutic Evaluation of Modified U1 snRNA Vectors in Human Fibroblasts}

\subsubsection{Modified U1 snRNA Vectors Transfection in Human Fibroblasts}

containing ampicillin $(100 \mu \mathrm{g} / \mathrm{mL})$ to a sterilized flask(s) (see Note 11) and innoculate all the bacterial growth from the miniculture(s). Incubate the flask(s) in an orbital shaking incubator at $37^{\circ} \mathrm{C}$ and $220 \mathrm{rpm}$ for $16-18 \mathrm{~h}$. Using an endotoxinfree maxiprep plasmid DNA purification kit, maxiprep the plasmid(s) containing the modified Ul snRNA construct (s) and perform its sequencing analysis for validation.

Even though we must always find a balance between the best possible experimental design and the resources available, adequate controls may never be forgotten. Still, there is a minimum standard for cell culture experiments that must always be met if we want to draw strong conclusions out of them. Therefore, adequate controls to the variables under test should always be included (see Note 14).

1. Grow both WT control and patient fibroblasts in T-75 flasks with DMEM + Glutamax medium supplemented with $10 \%$ FBS, $5 \%$ antibiotics, and $5 \%$ amphotericin $\mathrm{B}$, in an incubator at $37{ }^{\circ} \mathrm{C}$ with $95 \%$ humidity and $5 \% \mathrm{CO}_{2}$ following standard cell culture procedures.

2. On the day before transfection, detach the cells by trypsinization. Briefly, discard the growth medium and wash cells with $3 \mathrm{~mL}$ of PBS buffer. Then, discard the PBS and add $2 \mathrm{~mL}$ of trypsin-EDTA. Subsequently, incubate cells with the solution for $5 \mathrm{~min}$ at $37^{\circ} \mathrm{C}$. After this period, check in an inverted microscope that cells are detached and add $4 \mathrm{~mL}$ of fresh medium to inactivate trypsin-EDTA action.

3. Harvest the cells to a $15 \mathrm{~mL}$ tube and centrifuge at $500 \times g$ for 5 min to eliminate any traces of trypsin.

4. Discard the supernatant and resuspend cells in $4 \mathrm{~mL}$ of fresh medium.

5. Count cells in suspension with an hemocytometer (Neubauer chamber). Pipette a small volume of cell suspension (approximately $15 \mu \mathrm{L}$ ) to both hemocytometer chambers and count the cells present in all four external quadrants of each chamber by observing it in an inverted microscope. Considering the dimensions of the chamber $(1 \mathrm{~mm} \times 1 \mathrm{~mm} \times 0.1 \mathrm{~mm})$, each quadrant has a total volume of $0.1 \mathrm{~mm}^{3}$, which equals $10^{-4} \mathrm{~mL}$. Therefore, the total number of cells in the original suspension can be calculated with the following equation:

$$
N=\frac{\sum n}{8} \times 10^{4}
$$

where $N$ is the total number of cells per milliliter, $n$ is the number of cells counted in each quadrant of the Neubauer chamber and the $10^{4}$ factor allows for the correction of the total number of cells in $1 \mathrm{~mL}$ of cell suspension. 
6. For modified Ul snRNA vectors transfection, seed a total of $\sim 2.5-3 \times 10^{5}$ fibroblast cells into 6 -well plates and grow cells in DMEM + Glutamax medium supplemented with 10\% FBS, 5\% antibiotics and $5 \%$ amphotericin $\mathrm{B}$, in an incubator at $37{ }^{\circ} \mathrm{C}$ with $95 \%$ humidity and $5 \% \mathrm{CO}_{2}$.

7. On the next day (cells at $80-90 \%$ confluence), transfect the cells with quantities between 1 and $3.5 \mu \mathrm{g}$ of the modified Ul snRNA constructs using a transfection reagent according to the manufacturer's protocol (see Notes 15 and 16).

8. 24-48 h after transfection, harvest cells by trypsinization. Discard the growth medium of each plate well and wash cells with $1 \mathrm{~mL}$ of PBS buffer. Discard the PBS, add $500 \mu \mathrm{L}$ of trypsinEDTA to each well and incubate for $5 \mathrm{~min}$ at $37^{\circ} \mathrm{C}$. Then, check by microscopy that cells are rounding up and add $1 \mathrm{~mL}$ of DMEM + Glutamax medium to inactivate trypsin-EDTA. Harvest cells to $2 \mathrm{~mL}$ tubes and centrifuge at $500 \times g$ for $5 \mathrm{~min}$ at $4{ }^{\circ} \mathrm{C}$; discard the supernatant; wash cells with $1 \mathrm{~mL}$ of PBS buffer and centrifuge again. Proceed to RNA extraction or store the pellet(s) at $-80{ }^{\circ} \mathrm{C}$ for future use.

3.2.2 Analysis of Splicing Rescue by $R T-P C R$
1. Extract total RNA from the transfected human fibroblasts using a RNA extraction kit according to the manufacturer's protocol. Then, perform RNA quantification using a spectrophotometer.

2. For reverse transcription, use a cDNA synthesis kit following the manufacturer's protocol, and start with $1-2 \mu \mathrm{g}$ of total RNA. The cDNA synthesis reaction can be stored at $-20^{\circ} \mathrm{C}$ or used immediately for PCR amplification.

3. Perform a PCR in standard conditions using a Taq polymerase supplemented with its buffer, dNTPs, gene-specific primers for a final concentration of $0.4 \mu \mathrm{M}$ each (e.g. HGSNAT primers), $2 \mu \mathrm{L}$ of cDNA, and RNase free water to a final volume of $50 \mu \mathrm{L}$.

4. To evaluate the splicing rescue, analyze the amplification products through agarose gel electrophoresis in an agarose gel stained with $5 \mu \mathrm{L}$ of ethidium bromide (see Note 17). Choose a DNA ladder according to the size of the amplified band. After separation, visualize the gel using an UV transilluminator. As an example, Fig. I shows the results of the partial correction of HGSNAT exon 2 splicing after expression of a modified Ul snRNA (totally complementary to the $5^{\prime}$ ss of exon 2 ) in patients' fibroblasts.

5. Assess the identity of the obtained band(s) by sequencing analysis (see Note 18). For this purpose, purify the PCR products directly with a PCR clean-up kit if there is only one amplified band or when multiple bands are present excise each band from the gel and purify them using a gel band 
3.2.3 Assessment of the Effect of U1 snRNAInduced Splicing Rescue at Protein Level purification kit. Whatever the case, follow the indications present in the manufacturer's protocol.

6. Subject the purified bands to standard automated sequencing using gene-specific primers for the amplification (e.g. HGSNAT primers). Compare the obtained sequence (s) with the reference sequence of the gene of interest (retrieved from the Ensembl database) using the Clustal Omega bioinformatic tool (https://www.ebi.ac.uk/Tools/ $\mathrm{msa} /$ clustalo/), in order to analyze the effect of the modified Ul snRNA's in rescuing the normal splicing pattern.

While not included in this chapter, for it is case-specific, the effect of modified Ul snRNAs-treatment at protein level is mandatory whenever we want to proceed to in vivo studies in order to address the true therapeutic potential of a given Ul snRNA molecule.

Ideally, as soon as we get an RT-PCR pattern that confirms splicing correction to some extent, and that rescue is confirmed by band excision and Sanger sequencing, the overall effect of that rescue at protein level should also be checked. There is a variety of methods we can choose in order to address this issue, from the direct quantification of enzymatic activity (whenever the gene product under analysis has a catalytic activity) to that of the protein itself (through Western blot).

Usually, the method of choice depends on two major factors: the protein itself and the assays available in house to assess it. Virtually every method from Western blot to immunofluorescence may be informative and provide extra support to the conclusions drawn from the RT-PCR. Therefore, as a take-home message, we would recommend that, whenever designing Ul snRNA molecules for splicing rescue, the effect should be checked not only at cDNA level, but also at protein level.

\section{Notes}

1. This chapter is exclusively focused on mutation-adapted U1 snRNAs. Nevertheless, it is important to refer that there is a novel, second generation, of engineered Ul snRNAs, which may be used for therapeutic purposes: the so-called ExonSpecific Ul snRNAs (ExSpeUl). These ExSpeUls are complementary to non-conserved sequences downstream of mutant 5 'ss. In theory, ExSpeUl is expected to decrease the potential of off-target effects of UI snRNA-based therapies, while allowing for a single ExSpeUl to rescue multiple splicing defects that affect a single exon [4-6].

2. The pG3Ul vector [12] \{Susani, 2004, TCIRGl-dependent recessive osteopetrosis: mutation analysis, functional 
identification of the splicing defects, and in vitro rescue by $\mathrm{Ul}$ snRNA\} was used, but the human Ul snRNA sequence can be cloned in other standard expression vector(s).

3. Store LB-agar plates with antibiotics at $4{ }^{\circ} \mathrm{C}$ in the dark.

4. Mutagenic primers can be designed using the web-based QuikChange Primer Design Program, available online at www. agilent.com/store/primerDesignProgram.jsp (we recommend to read the "help" section of the program). However, it is important to take into account a number of considerations:

(a) both mutagenic primers must contain the desired mutation(s) and anneal to the same sequence on opposite strands of the plasmid;

(b) each primer should have between 25 and 45 bases in length with a melting temperature $(\mathrm{Tm})$ of $\geq 78{ }^{\circ} \mathrm{C}$;

(c) the desired mutation(s) should be located in the middle of the primer $(\sim 12-15$ nucleotides of the correct sequence on both sides);

(d) the primers should have a minimum GC content of $40 \%$ and should terminate in one or more $\mathrm{C}$ or $\mathrm{G}$ bases;

(e) the primers do not need to be $5^{\prime}$ phosphorylated and purification may either be performed by liquid chromatography (HPLC) or by polyacrylamide gel electrophoresis (PAGE).

5. To modify the pG3Ul we recommend to use the QuikChange $^{\text {TM }}$ II mutagenesis kit (Agilent). However, other sitedirected mutagenesis commercial kits can be used. The kit should be chosen according to the plasmid length and the type of mutations to introduce.

6. According to the type of mutation(s) to be inserted in the Ul snRNA WT sequence, the number of PCR cycles varies. For point mutations ( 1 nucleotide change) use 12 cycles; for single aminoacid changes ( 3 nucleotides) use 16 cycles and for multiple amino acid deletions or insertions ( $\geq 4$ nucleotides) use 18 cycles.

7. The number $(n)$ of min of PCR extension step recommended is usually $1 \mathrm{~min} / \mathrm{kb}$. However, using the QuikChange ${ }^{\mathrm{TM}}$ II mutagenesis kit (Agilent) we usually increment the time for $2 \mathrm{~min} /$ $\mathrm{kb}$. For the pG3Ul plasmid length, 8 min should work, but from our experience adding one more min to this step (in this case $9 \mathrm{~min}$ for extension) gives the best results.

8. If a thermomixer is not available, follow the site-directed mutagenesis kit manufacturer's recommendations concerning shaking of transformation reactions. 
9. The entire volume of transformation reaction can be plated on a single LB-agar plate. However, depending on the transformation efficiency this may originate a huge number of colonies which are then difficult to select. Therefore, we recommend to use more than one plate and spread different volumes to increase the probability to obtain individualized colonies (e.g. 200 and $100 \mu \mathrm{L}$ ). When plaquing lower volumes a small quantity $(1: 1)$ of SOC medium can be added to the transformation reaction to dilute and help to spread the transformation product.

10. If colonies cannot be selected immediately, store plate(s) at $4{ }^{\circ} \mathrm{C}$.

11. The total volume of the tube should allow a volume of air that is $5 \times$ the volume of LB medium (e.g. $3 \mathrm{~mL}$ of LB medium in a $15 \mathrm{~mL}$ tube; $5 \mathrm{~mL}$ of LB medium in a $25 \mathrm{~mL}$ tube, etc.).

12. Before starting the miniprep(s) procedure, a sample of bacterial culture can be preserved in a "glycerolate" for future use. For a final volume of $1 \mathrm{~mL}$, add a part of bacterial culture and a part of sterilized glycerol to a $2 \mathrm{~mL}$ tube for a final concentration of $\sim 10-15 \%$ of glycerol. Vortex immediately and store at $-80^{\circ} \mathrm{C}$.

13. To avoid the need to pick another bacterial colony from an LB-agar plate, the glycerolate(s) (see Note 12) can be used to prepare a new miniculture. Briefly, defrost the glycerolate on ice, scrape it lightly with a pipette tip or aspirate few microliters and pipet them up and down into a tube containing the desired volume of LB medium and ampicillin $(100 \mu \mathrm{g} / \mathrm{mL})$. Incubate the tube(s) in an orbital shaking incubator as recommended in step 4 of Subheading 3.1.1.

14. In the transfection experiments here referred (see step 7 of Subheading 3.2.1) we included two negative controls: one where only the transfection reagent was added to the cells and other where the minigene expressing the WT Ul sequence was transfected on cells.

15. For liposome-based transfection of fibroblasts, Lipofectamine ${ }^{\circledR}$ 2000 (Invitrogen) or other commercial lipofection reagent can also be tested. To further increase transfection efficacy, the modified Ul's can also be inserted into the cells by the electroporation technique. For both methods we recommend to optimize the amount of transfection reagent according to the quantity of modified Ul and number of cells to transfect.

16. To assess transfection efficiency, transfect fibroblasts with a control plasmid encoding GFP or RFP and monitor fluorescence by microscopy. Also, the cell uptake of the modified Ul's can be confirmed by PCR with specific primers ( $\mathrm{Ul} \mathrm{Fw}-5^{\prime} \mathrm{A}$ TCGAAATTAATACGACTCA $3^{\prime}$ and Ul Rv-5' CTGGGA AAACCACCTTCGT $3^{\prime}$ ). Otherwise, clone the WT human U1 
snRNA cassette from pG3Ul vector in a plasmid encoding GFP and monitor fluorescence and Ul expression simultaneously.

17. Adjust the agarose gel percentage according to the molecular weight of the target amplified products.

18. In RT-PCR analysis after Ul snRNA's transfection, the size of the amplified band(s) seen on the agarose gel can give an idea of whether the aberrantly spliced exon under study is included in the cDNA or not. However, it is necessary to sequence the amplified band(s) from control and patient fibroblasts treated with the different modified Ul snRNAs, to confirm the correct splicing pattern.

\section{Acknowledgments}

The authors would like to acknowledge Prof. Dr. Belén Pérez (Molecular Biology Department, Faculty of Sciences, University Autonoma of Madrid, Spain) for kindly provide the pG3Ul vector. In addition, the authors would like to acknowledge BioMedCentral, Part of Springer Nature for allowing the reproduction in this chapter of an adapted version of a figure originally published in Matos, L. et al. Therapeutic strategies based on modified Ul snRNAs and chaperones for Sanfilippo C splicing mutations. Orphanet J Rare Dis 9, 180 (2014). https://doi.org/10.1186/ s13023-014-0180-y. Copyright (C) 2014, Springer Nature. This work was partially supported by Fundação para a Ciência e Tecnologia (FCT) IP (project: FCT/PTDC/BBBBMD/6301/2014) and by The National MPS Society (project: 2019DGH1642).

\section{References}

1. van der Feltz C, Anthony K, Brilot A, Pomeranz Krummel DA (2012) Architecture of the spliceosome. Biochemistry 51(16): 3321-3333. https://doi.org/10.1021/ bi201215r

2. Buratti E, Baralle D (2010) Novel roles of U1 snRNP in alternative splicing regulation. RNA Biol 7(4):412-419

3. Carmel I, Tal S, Vig I, Ast G (2004) Comparative analysis detects dependencies among the $5^{\prime}$ splice-site positions. RNA 10(5):828-840. https://doi.org/10.1261/rna.5196404

4. Pinotti M, Bernardi F, Dal Mas A, Pagani F (2011) RNA-based therapeutic approaches for coagulation factor deficiencies. J Thromb Haemost 9(11):2143-2152. https://doi.org/10. $1111 /$ j.1538-7836.2011.04481.x
5. Coutinho MF, Matos L, Santos JI, Alves S (2019) RNA therapeutics: how far have we gone? Adv Exp Med Biol 1157:133-177. https://doi.org/10.1007/978-3-03019966-1_7

6. Hwu WL, Lee YM, Lee NC (2017) Gene therapy with modified Ul small nuclear RNA. Expert Rev Endocrinol Metab 12(3): 171-175. https://doi.org/10.1080/ 17446651.2017 .1316191

7. Balestra D, Faella A, Margaritis P, Cavallari N, Pagani F, Bernardi F, Arruda VR, Pinotti M (2014) An engineered Ul small nuclear RNA rescues splicing defective coagulation F7 gene expression in mice. J Thromb Haemost $12(2)$ : 177-185. https://doi.org/10.1111/jth. 12471 
8. Lee NC, Lee YM, Chen PW, Byrne BJ, Hwu WL (2016) Mutation-adapted Ul snRNA corrects a splicing error of the dopa decarboxylase gene. Hum Mol Genet 25(23):5142-5147. https://doi.org/10.1093/hmg/ddw323

9. Lee B, Kim YR, Kim SJ, Goh SH, Kim JH, Oh SK, Baek JI, Kim UK, Lee KY (2019) Modified Ul snRNA and antisense oligonucleotides rescue splice mutations in SLC26A4 that cause hereditary hearing loss. Hum Mutat 40(8): 1172-1180. https://doi.org/10.1002/ humu. 23774

10. Breuel S, Vorm M, Bräuer AU, OwczarekLipska M, Neidhardt J (2019) Combining engineered Ul snRNA and antisense oligonucleotides to improve the treatment of a BBSI splice site mutation. Mol Ther Nucl Acids 18: 123-130. https://doi.org/10.1016/j.omtn. 2019.08.014
11. Matos L, Canals I, Dridi L, Choi Y, Prata MJ, Jordan P, Desviat LR, Pérez B, Pshezhetsky AV, Grinberg D, Alves S, Vilageliu L (2014) Therapeutic strategies based on modified $\mathrm{Ul}$ snRNAs and chaperones for Sanfilippo C splicing mutations. Orphanet J Rare Dis 9:180. https://doi.org/10.1186/s13023-014$0180-y$

12. Susani L, Pangrazio A, Sobacchi C, Taranta A, Mortier G, Savarirayan R, Villa A, Orchard P, Vezzoni P, Albertini A, Frattini A, Pagani F (2004) TCIRGl-dependent recessive osteopetrosis: mutation analysis, functional identification of the splicing defects, and in vitro rescue by Ul snRNA. Hum Mutat 24(3):225-235. https://doi.org/10.1002/humu.20076

13. Lund E, Dahlberg JE (1984) True genes for human Ul small nuclear RNA. Copy number, polymorphism, and methylation. J Biol Chem 259(3):2013-2021

Open Access This chapter is licensed under the terms of the Creative Commons Attribution 4.0 International License (http://creativecommons.org/licenses/by/4.0/), which permits use, sharing, adaptation, distribution and reproduction in any medium or format, as long as you give appropriate credit to the original author(s) and the source, provide a link to the Creative Commons license and indicate if changes were made.

The images or other third party material in this chapter are included in the chapter's Creative Commons license, unless indicated otherwise in a credit line to the material. If material is not included in the chapter's Creative Commons license and your intended use is not permitted by statutory regulation or exceeds the permitted use, you will need to obtain permission directly from the copyright holder. 